\title{
Concomitant protective and therapeutic role of verapamil in chronic mercury induced nephrotoxicity in the adult rat: histological, morphometric and ultrastructural study
}

Nabila Yousef Abdel Haleem, Hoda Mahmoud El-Aasar, Sherif Mohamed Zaki, Sherif Mohamed Sabry, Ahmed Wafiq El-Zainy

Department of Anatomy, Faculty of Medicine, Cairo University, Cairo, Egypt

Submitted: 19 May 2012

Accepted: 9 November 2012

Arch Med Sci 2015; 11, 1: 199-209

DOI: 10.5114 /aoms.2013.37342

Copyright (c) 2015 Termedia \& Banach

\section{Abstract}

Introduction: Mercury intoxication is a widespread problem as mercury is used in the manufacture of thermometers, batteries and electrical switches. It forms one of the most diffusible environmental pollutants. Mercury has a nephrotoxic effect which could occur at low exposure levels. Verapamil could help in the treatment of mercuric toxicity. The aim of the study was to examine the protective and therapeutic effect of concomitant verapamil on chronic mercuric chloride nephrotoxicity. This was done through histological, morphometric and transmission electron microscopic studies.

Material and methods: Sixty adult male albino rats were used. The rats were divided into a control group and 4 experimental groups: group I $\left(\mathrm{HgCl}_{2}\right)$, group II (concomitant $\mathrm{HgCl}_{2}$ and verapamil), group III $\left(\mathrm{HgCl}_{2}\right.$ withdrawal) and group IV ( $\mathrm{HgCl}_{2}$ withdrawal then verapamil treatment).

Results: Chronic administration of $\mathrm{HgCl}_{2}$ resulted in cortical nephrotoxic effects in the form of glomerular sclerosis, acute tubular necrosis and interstitial inflammatory cellular infiltration which eventually ended in interstitial fibrosis. Concomitant use of verapamil with $\mathrm{HgCl}_{2}$ improved the previous pathological changes partially. The findings in group III were less severe compared to group IV. The persistence of the pathological findings in these groups reflects the irreversible nephrotoxic changes caused by chronic $\mathrm{HgCl}_{2}$ exposure.

Conclusions: We concluded that the concomitant administration of verapamil has a much better effect in minimizing the nephrotoxic effect caused by chronic $\mathrm{HgCl}_{2}$ than its therapeutic administration. So, we recommended the prophylactic use of verapamil in suspected cases of chronic mercuric chloride nephrotoxicity to preserve renal function.

Key words: verapamil chronic mercury nephrotoxicity.

\section{Introduction}

Mercury intoxication is a common problem in Egypt; for instance, dentists and dental nurses have significant exposure to mercury vapour [1]. In one study, which included tailing from a gold mine, at Allaqi Wadi Aswan, Egypt, there was high contamination of the soil and plants with toxic metals $(\mathrm{Hg}, \mathrm{Cd}, \mathrm{Pb}$ and $\mathrm{As}$ ), and the author recommended not using the plants and soil for grazing or agriculture [2]. The mercury intoxication problem is widespread all over the world as mercury is used in manufacturing thermometers, batteries and electrical switches [3] as well as in fungicides and pesticides [4]. Mercury is also present in skin lightening creams and

\author{
Corresponding author: \\ Sherif Mohamed Zaki MD \\ Anatomy Department \\ Faculty of Medicine \\ Cairo University \\ El-Kasr Al Aini Street \\ Cairo, Egypt \\ Phone: +20226706317, \\ $+201227457431$ \\ E-mail: zaky.sherif@yahoo.com
}


hair-dyeing agents [5]. It forms one of the most diffusible environmental pollutants to which humans are exposed [6].

It is well known that mercury has a nephrotoxic effect [7] which can occur at low exposure levels [8]. This toxicity affects primarily the proximal convoluted tubules (PCT) $[9,10]$.

Depending on the effect of mercury on calcium homeostasis, verapamil could help in the treatment of mercuric toxicity [11]. It produces a significant improvement in renal function in treatment of mercury-induced nephrotoxicity [12]. It also prevented acute mercury chloride nephrotoxicity [13].

Whether concomitant use of verapamil with exposure to chronic mercuric chloride $\left(\mathrm{HgCl}_{2}\right)$ has total protection on the kidney is a relevant question. The other important question is whether verapamil treatment has a similar role to its protective role in chronic mercuric chloride nephrotoxicity. So, the aim of our study was to examine the effect of the concomitant protective and therapeutic role of verapamil on chronic mercuric chloride nephrotoxicity in adult male rats. This was done through histological, morphometric and transmission electron microscopic studies (TEM).

\section{Material and methods}

Sixty adult male Sprague Dawley rats (Animal House, Faculty of Medicine, Cairo University), weighing 250-300 g, were used. Five animals were housed per cage, and the animals were acclimatized to standard laboratory conditions (12: 12-hour light-dark cycle, temperature $20^{\circ} \mathrm{C}$, fed ad libitum and allowed free water supply). Only male rats were used in this study to exclude possible sex differences.

The experiment was performed according to the Helsinki agreement on the guiding principles for research involving animals and human beings. The current study was reviewed by the ethical committee of experiments on animals, Faculty of Medicine, Cairo University. The committees approved the current work.

The animals were divided into control and experimental groups.

\section{Control group}

This group was subdivided into two subgroups; each one consisted of ten rats. Subgroup a: The rats were sacrificed after 30 days. Subgroup b: The rats were sacrificed after 60 days.

\section{Experimental group}

This group was subdivided into four groups; each group consisted of ten rats. Group I: the rats received $\mathrm{HgCl}_{2}$ daily for 30 days. They were sacrificed 1 day after the last injection. Group II: the rats received both $\mathrm{HgCl}_{2}$ and verapamil daily for 30 days. They were sacrificed 1 day after the last injection. Group III $\left(\mathrm{HgCl}_{2}\right.$ withdrawal): The rats were given $\mathrm{HgCl}_{2}$ daily for 30 days. They were sacrificed 30 days after the last injection. Group IV $\left(\mathrm{HgCl}_{2}\right.$ withdrawal then verapamil treatment): The rats received $\mathrm{HgCl}_{2}$ daily for 30 days. Then they were given verapamil alone for another 30 days. They were sacrificed one day after the last injection.

Mercuric chloride was obtained in powder form from Algumhoria Pharm. and Chem. Co. It was dissolved in distilled water and was given subcutaneously in a dose of $0.02 \mathrm{mg} / \mathrm{kg}$. This is a nephrotoxic dose for the adult Sprague-Dawley rats [14].

Verapamil hydrochloride was obtained from Kahira Pharm. and Chem. Co. under license from Abbott Laboratories. It was given subcutaneously at a dose of $0.5 \mathrm{mg} / \mathrm{kg}$ [15].

The animals were sacrificed by a high dose of ether. Both kidneys were dissected and removed.

\section{Light microscopic study}

Parts of the obtained specimen were fixed in buffered formol saline, processed for paraffin sections of $5 \mu \mathrm{m}$ thickness, and sections were stained with haematoxylin and eosin $(H+E)$, Masson's trichrome and periodic acid-Schiff (PAS) stains for histological study [16]. The prepared sections were all examined and photographed using a Canon digital camera (Canon, Japan), attached to an IBM computer system.

\section{Ultrastructural study}

The other parts of obtained specimens were prepared for TEM [17]. Examination and photography of specimens were carried out using a Jeom-1400 transmission electron microscope (JEOL Ltd./Japan); the examination was done in the electron microscopy department, Faculty of Agriculture, Cairo University.

\section{Image analyser study}

The sections were examined using Leica Qwin 500 image analysis software on an IBM operated computer system (Leica Microsystems, Wetzlar, Germany).

Four specimen preparations from each rat (with a total of 40 preparations in each experimental subgroup) were subjective to quantitative studies:

The optical density of the basement membrane of renal corpuscles, basement membrane and brush border of the PCT with PAS reaction were studied. This was done by transforming the image into a grey image and then masking the positive areas with blue binary colour. The parameters chosen were the number of pixels, sum of grey and mean grey. The optical density was expressed in the form 

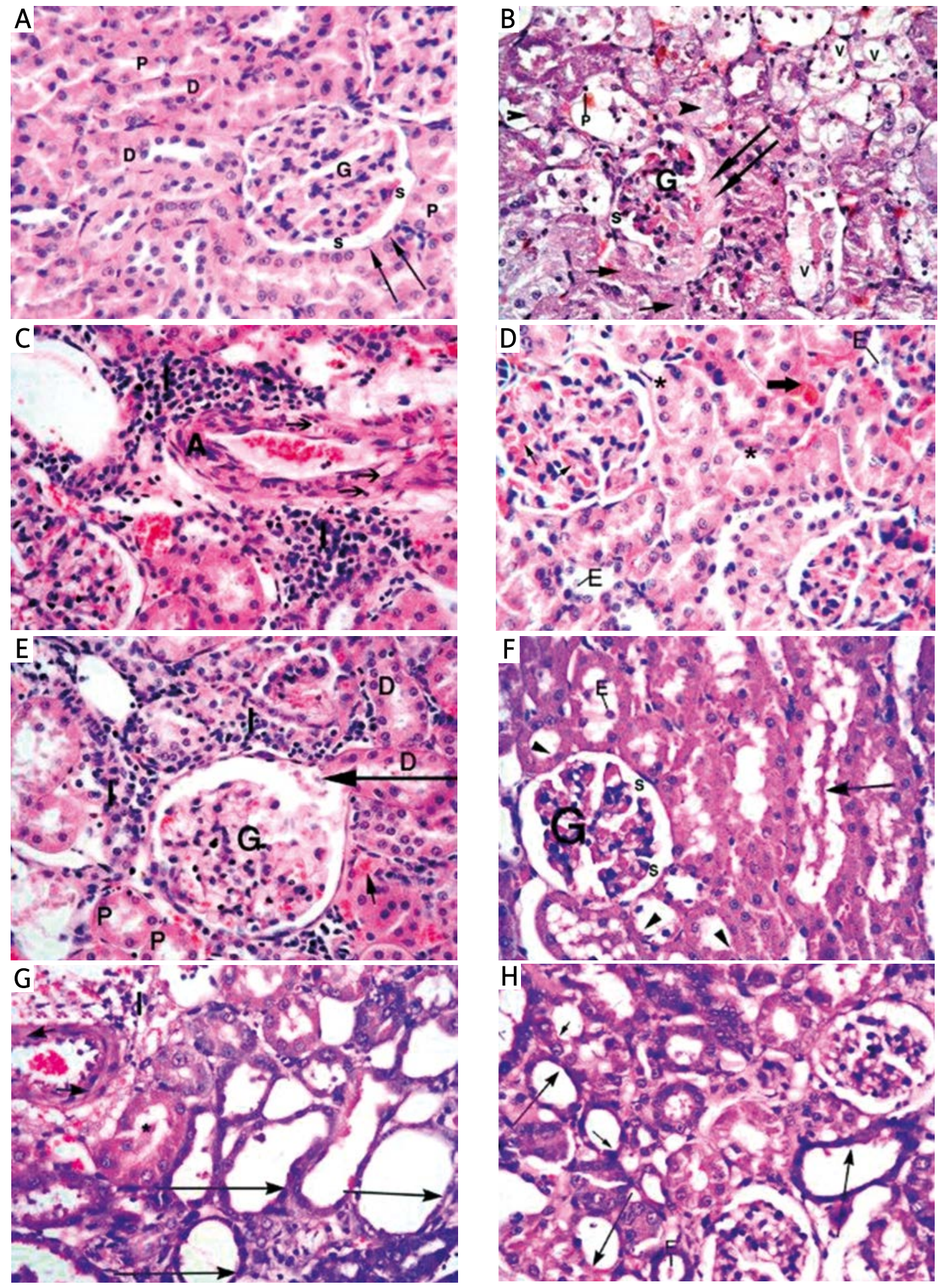

Figure 1 A - Normal glomerulus (G), parietal layer of Bowman's capsule (arrows) and urinary spaces in a control rat. Note normal PCT (P) and DCT (D) (H + E; 400x). B - Interstitial exudate (short arrows), shrunken glomerulus (G) with fluid exudate (long arrows) filling the urinary spaces. Cytoplasmic vacuolation $(V)$, pyknotic nucleus (p) with intra-luminal cast formation (arrowhead) of the tubular epithelium in a rat of group I ( $\mathrm{H}+\mathrm{E} ; 400 \mathrm{x})$. C - Thickened renal arteriole (A) with intramuscular vacuolation (short arrows) and interstitial cellular infiltration (I) in a rat of group I ( $H+E ; 400 x)$. D - Glomerular (thin arrows) and interstitial (thick arrow) congestion in a rat of group II. Some tubules are normally $\left(^{*}\right)$ and others showed intra-luminal exfoliation of the epithelial cells (E) (H+E; 400x). E - Interstitial cellular infiltration (I), capillary congestion (short arrow) and tissue debris (long arrow) in the urinary space of a glomerulus (G) in a rat of group II. Both PCT (P) and DCT (D) are normal (H + E; 400x). F- Congested glomerulus (G) with wide urinary space (s). PCT exhibited partial loss of the apical brush border (arrowheads), intraluminal exfoliation of the tubular cells (E) and cast formation (arrow) in a rat of group III ( $\mathrm{H}+\mathrm{E} ; 400 \times)$. G - Dilated renal tubules with flattening of the epithelium (long arrows) with intraluminal cast formation (*), thickened renal arteriole (short arrow) and interstitial cellular infiltration (I) in a rat of group III (H + E; 400x). $\mathrm{H}$ - Dilation of some renal tubules with flattening (long arrows), vacuolation (short arrows) and intraluminal exfoliation (E) of their epithelium in a rat of group IV (H+E; 400x) 

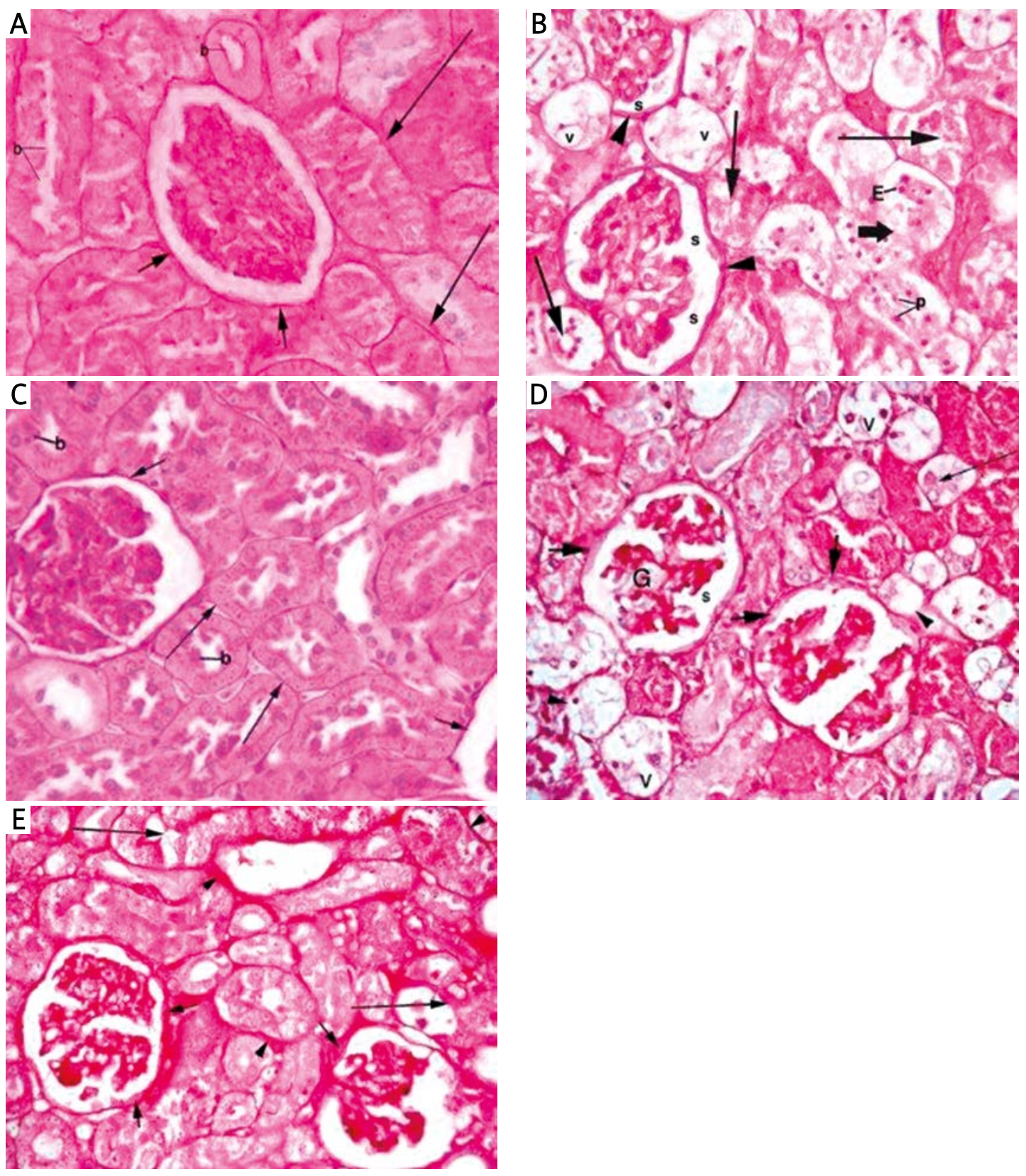

Figure 2. A - Parietal layer of Bowman's capsule (short arrows), basement membrane (long arrows) and apical brush border (b) of the renal tubules of a control rat (PAS; 400x). B - Increase in the thickness of the parietal layer of Bowman's capsule (arrowheads), widening of the urinary spaces (s), partial interruption of the tubular basement membrane (thick arrow), complete loss of the apical brush border (long arrows), vacuolations (v), intraluminal exfoliation (E) and pyknotic nuclei (p) of the tubular epithelium in a rat of group I (PAS; 400x). C - Thin parietal layer of Bowman's capsule (short arrows), partial loss of the apical brush border (b) and intact basement membrane (long arrows) of PCT in a rat of group II (PAS; 400x). D - Focal thickening of the parietal layer of Bowman's capsule (short arrows) with shrunken glomeruli $(\mathrm{G})$, and widening of the urinary space $(\mathrm{s})$. Pyknosis $(\mathrm{p})$ and vacuolations $(\mathrm{V})$ of the tubular epithelial lining with partial loss of the apical brush border (arrow) and the tubular basement membranes (arrowheads) in a rat of group III (PAS; 400x). E-Thickening of the tubular basement membrane (arrowheads) and the parietal layer of Bowman's capsule (short arrow) with partial loss of the apical brush border (long arrows) of renal tubular cells in a rat of group IV (PAS; 400×)

of mean grey that was obtained by the following equation: mean grey $=$ no. of pixels/sum of grey.

The area percentage of collagen fibres in Masson's trichrome stained sections was measured inside a standard measuring frame of a known area.
The collagen fibres were selected and masked with blue binary colour. Then the area of the blue binary colour was measured and expressed as an area percentage in relation to the area of the standard measuring frame. 
Table I. Mean and SD of area \% of collagen, PAS reaction, and area of urinary space of the different groups

\begin{tabular}{|ccccc|}
\hline Group & $\begin{array}{c}\text { Number of examined } \\
\text { preparations }\end{array}$ & $\begin{array}{c}\text { Area \% preparations } \\
\text { in each group }\end{array}$ & $\begin{array}{c}\text { PAS reaction } \\
\text { of collagen }\end{array}$ & $\begin{array}{c}\text { Area of urinary } \\
\text { space }\end{array}$ \\
\cline { 2 - 5 } & 40 & Mean \pm SD & Mean \pm SD & $2105.2 \pm 687.9$ \\
\hline Control & 40 & $4.6 \pm 2.2$ & $0.48 \pm 0.03$ & $2719.8 \pm 760.6$ \\
\hline II & 40 & $24.6 \pm 7$ & $0.32 \pm 0.02$ & $2301.5 \pm 451.7$ \\
\hline III & 40 & $17.4 \pm 7.5$ & $0.43 \pm 0.02$ & $2506.8 \pm 692.5$ \\
\hline IV & 40 & $23.4 \pm 3.8$ & $0.34 \pm 0.03$ & $2445.5 \pm 537.2$ \\
\hline
\end{tabular}

Table II. Comparison of mean area \% of collagen, mean of PAS reaction, and mean area of urinary space of the different groups

\begin{tabular}{|c|c|c|c|c|c|}
\hline Parameter & Group & Versus group & Mean difference & Value of $p$ & $F$ ratio \\
\hline \multirow[t]{4}{*}{ Mean area $\%$ of collagen } & \multirow[t]{2}{*}{ Control } & I & -19.9 & $0.001^{\star *}$ & \multirow[t]{4}{*}{30.9} \\
\hline & & II & -12.8 & $0.001^{* *}$ & \\
\hline & I & II & 7.1 & $0.002^{\star *}$ & \\
\hline & III & IV & 2.8 & 0.2 & \\
\hline \multirow{4}{*}{$\begin{array}{l}\text { Mean optical density } \\
\text { of PAS reaction }\end{array}$} & \multirow[t]{2}{*}{ Control } & I & 0.16 & $0.001^{* *}$ & \multirow[t]{4}{*}{44.4} \\
\hline & & II & 0.05 & $0.002^{\star *}$ & \\
\hline & 1 & II & -0.1 & $0.001^{\star *}$ & \\
\hline & III & IV & 0 & 0.9 & \\
\hline \multirow{4}{*}{$\begin{array}{l}\text { Mean area of urinary } \\
\text { space }\end{array}$} & \multirow[t]{2}{*}{ Control } & 1 & -614.5 & $0.03^{*}$ & \multirow[t]{4}{*}{1.6} \\
\hline & & II & -196.3 & 0.5 & \\
\hline & I & II & 418.2 & 0.1 & \\
\hline & III & IV & 61.3 & 0.8 & \\
\hline
\end{tabular}

*Statistically significant.

The mean area of the urinary space in sections stained with $\mathrm{H}+\mathrm{E}$ were examined within the standard measuring frame of a known area. Using the binary edit, areas of glomerular tufts and Bowman's capsule were masked with a green colour and the mean values were obtained. The difference between the Bowman's capsule and glomerular tuft areas corresponds to the area of Bowman's (urinary) space.

\section{Statistical analysis}

The data were collected and studied using analysis of variance (two-tailed ANOVA) to compare between the different groups using SPSS 20 statistical program. The quantitative data were examined by the Kolmogorov-Smirnov test for normality. The level of significance was considered at $p$-value $<0.05$.

\section{Results}

\section{Results of $\mathrm{H}+\mathrm{E}$ stained sections}

Histological examination of the kidney of the control rats exhibited normal architecture of the glomeruli and renal tubules.
The cortex of the rats of group I exhibited the presence of shrunken glomeruli with fluid exudate filling the urinary spaces. Degeneration of renal tubules was observed with different forms such as cytoplasmic vacuolation of the tubular epithelial, pyknotic nuclei, intra-luminal exfoliation and cast formation with the presence of interstitial exudate. Also, thickening of the renal arterioles with intramuscular vacuolation and interstitial cellular infiltration in their lumens were observed.

With concomitant use of verapamil (group II) much improvement was observed compared to group I. This improvement was partial as there were glomerular congestion, interstitial cellular infiltration and intra-luminal tubular exfoliation of the epithelial cells.

The mercuric chloride withdrawal group (group III) has the same pathological findings as group I. Glomeruli congestion, widening of the urinary spaces, partial loss of the PCT brush border with intraluminal exfoliation of the tubular cells, thickening of the renal arterioles and interstitial cellular infiltration are observed. These findings were slightly attenuated in group IV (Figure 1). 


\section{Results of PAS stained sections}

Increase in the thickness of the parietal layer of Bowman's capsules with complete loss of the apical brush border of the renal tubules and partial interruption of their basement membranes were observed in the rats of group $\mathrm{I}$.

Comparing group II to the previous experimental group (group I), much improvement of the pre- vious pathological findings were observed. The parietal layer of Bowman's capsules became thin, the loss of the apical brush borders became partial and the tubular basement membranes were intact.

Both groups III and IV had thickening of the parietal layer of Bowman's capsules and the tubular basement membranes. The apical brush borders of the renal tubules were partially lost. These findings
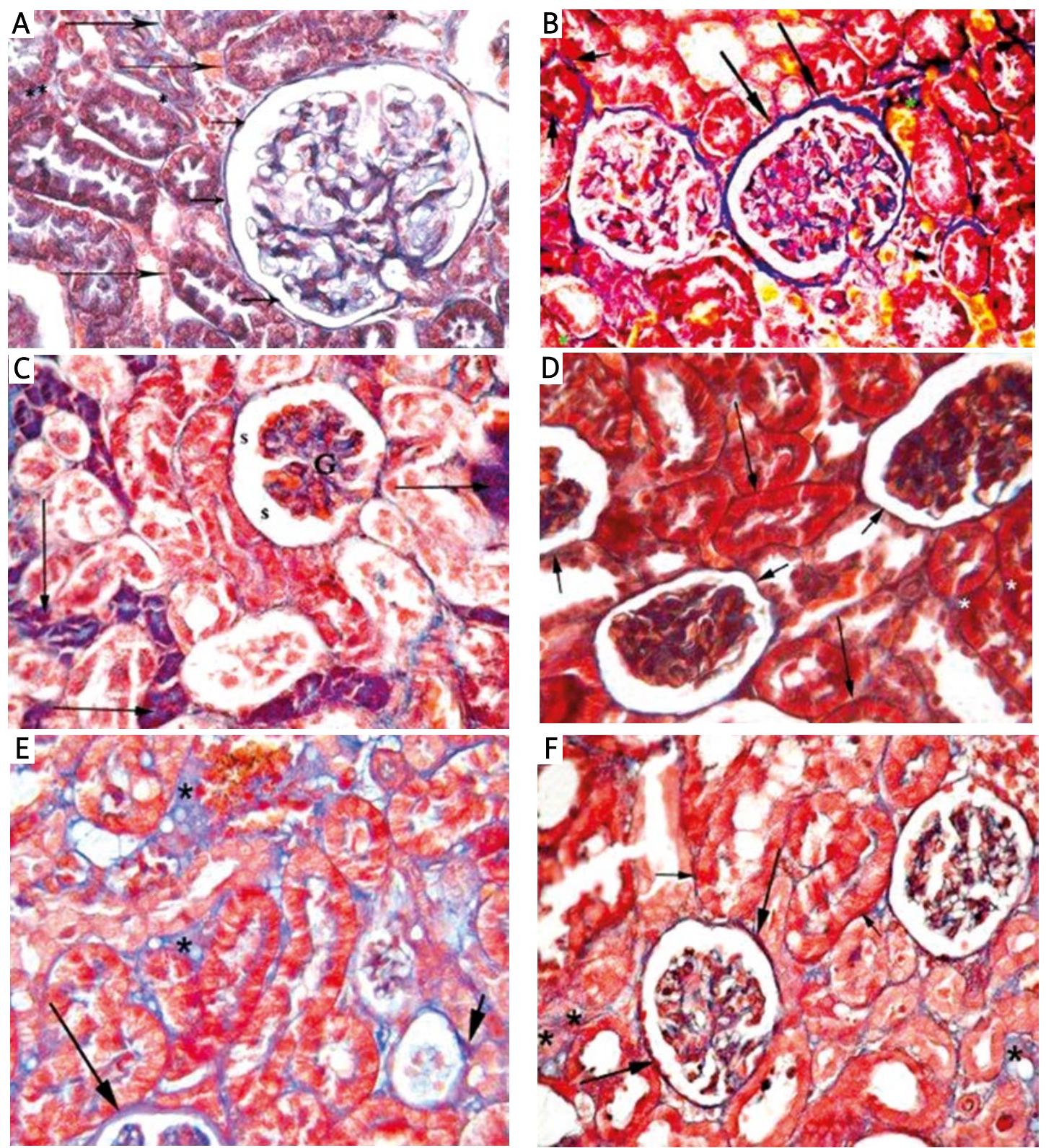

Figure 3. A - Parietal layer of Bowman's capsule (short arrows), tubular basement membrane (long arrows) and interstitial connective tissue ${ }^{*}$ ) in a control rat (Masson's trichrome; 400x). B - Thickening of the parietal layer of Bowman's capsule (long arrows) and the basement membrane (short arrows) of renal tubules with focal areas of increased interstitial fibrous tissue formation (*) in a rat of group I (Masson's trichrome; 400x). C - Increase in the interstitial fibrous tissue formation (arrows), widening of the urinary spaces and shrinkage of the glomeruli (G) in a rat of group I (Masson's trichrome; 400x). D- Thinning of the parietal layer of Bowman's capsule (short arrows) and the tubular basement membrane (long arrows). Note the presence of interstitial fibrous tissue $\left({ }^{*}\right)$ in a rat of group II (Masson's trichrome; 400x). E- Focal areas of increased interstitial fibrous tissue formation $\left({ }^{*}\right)$, thickening of the parietal layer of Bowman's capsule (long arrow) and the tubular basement membrane in a rat of group III (Masson's trichrome; 400x). F- Increased interstitial fibrous tissue formation $\left(^{*}\right)$ with focal thickening of both the parietal layer of Bowman's capsule (long arrows) and the tubular basement membrane (short arrows) in a rat of group IV (Masson's trichrome; 400x) 
were less severe in group IV compared to group III (Figure 2).

\section{Results of Masson's trichrome stained sections}

Thickening of the parietal layer of Bowman's capsules and the tubular basement membranes were observed in the rats of group I when compared to the control group. Focal areas of increased interstitial fibrous tissue formation were a constant finding in this group. Comparing group II to group I, all of the previous pathological findings became much more attenuated.
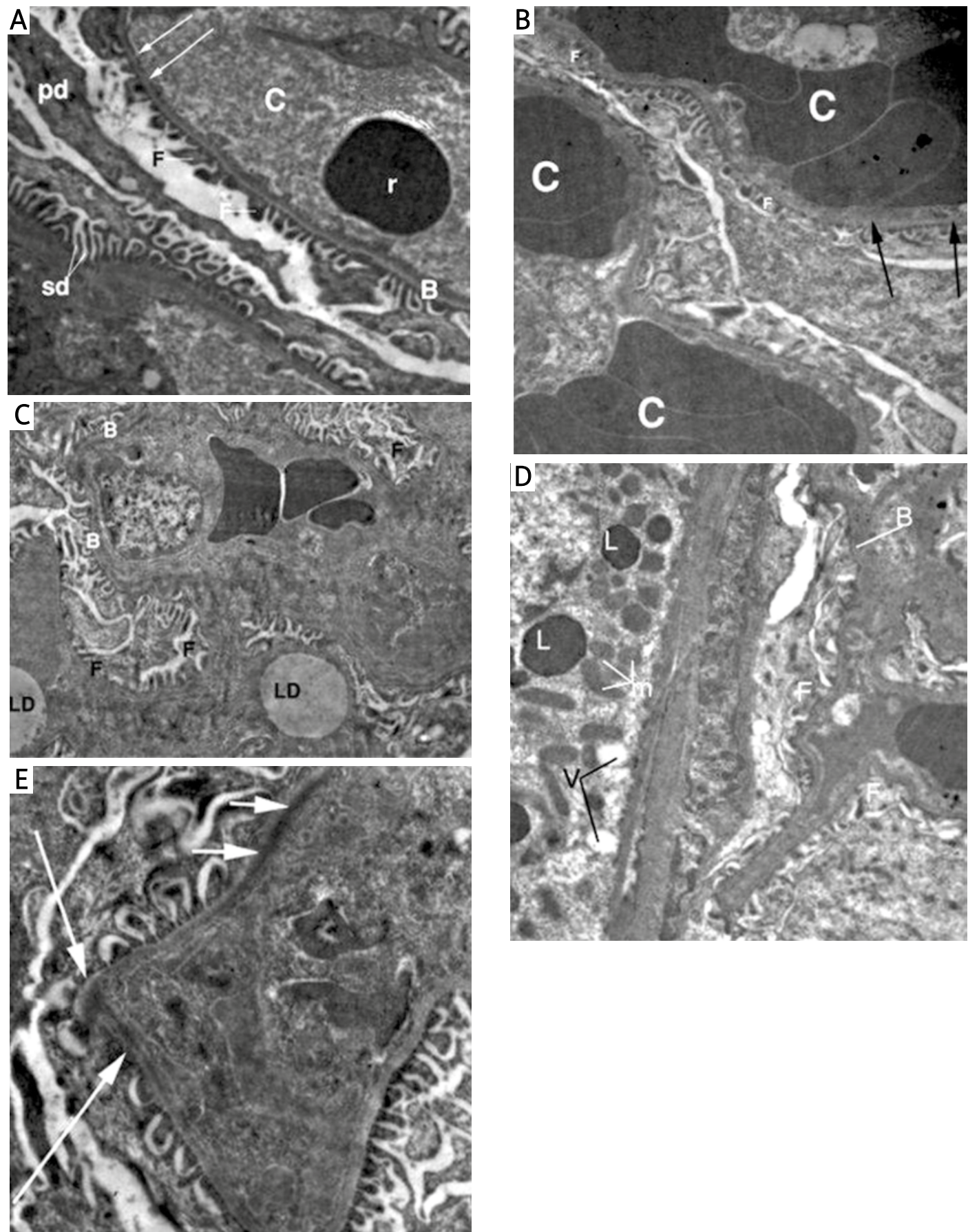

Figure 4. A - Trilaminar appearance of the glomerular basement membrane (B) fenestrated capillary endothelial lining (arrows) with a red blood cell ( $r$ ) in the capillary lumen (C), discrete foot process (F) of the podocytes (pd) separated by slit diaphragms (sd) in a control rat (5000x). B - Intraglomerular capillary congestion (C), thickened glomerular basement membrane (arrows) with fusion (effacement) of the foot processes (F) of the podocytes obliterating slit diaphragms between them in a rat of group I (6000x). C - Normal appearance of the glomerular basement membrane (B), focal fusion of the foot processes (F) and intraglomerular lipid droplets (LD) in a rat group II (4000x). D - Focal thickening of the glomerular basement membrane (B), fusion of the foot processes (F) of the podocytes, basally located mitochondria $(\mathrm{m})$, vacuolations $(\mathrm{V})$ and lysosomes $(\mathrm{L})$ of variable size in PCT in a rat of group III (5000x). E - Loss of the trilaminar appearance of the glomerular basement membrane (short arrows) and occasional fusion (long arrows) of the foot processes of the podocytes in a rat of group IV (10000x) 

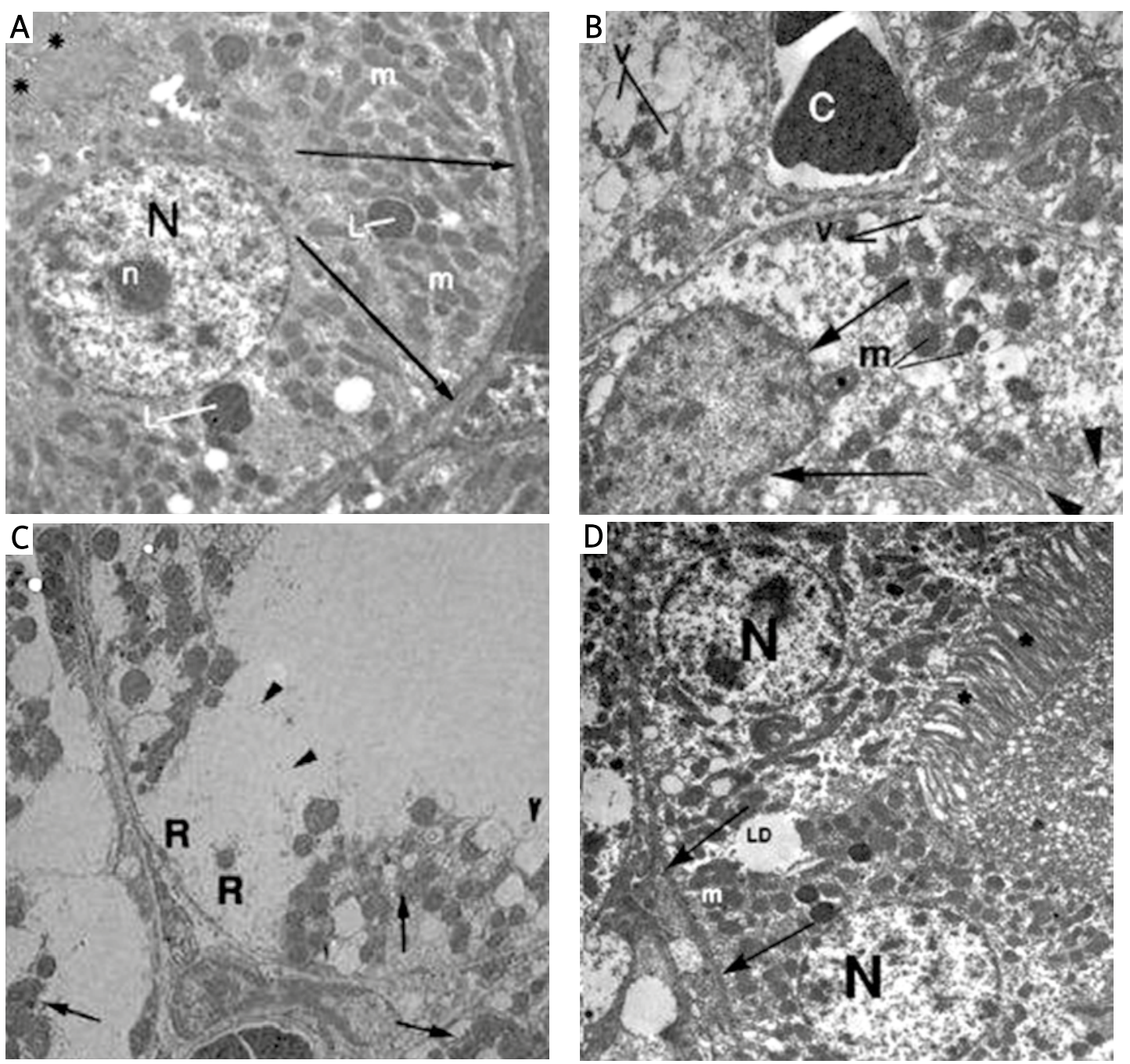

Figure 5. A - PCT with epithelial lining with rounded mid-positioned nucleus $(\mathrm{N})$, nucleolus (n), lysosomes (L), apical microvilli (*), abundant mitochondria (m) and basal cell membrane (long arrows) in a control rat (3000x). B-PCT with irregular nuclear membrane, margination of chromatin material (arrows), complete loss of the apical microvilli (arrowheads), dilated interstitial capillary (C), basal aggregation of mitochondria $(\mathrm{m})$ and vacuolations $(\mathrm{V})$ in a rat of group I (5000x). C - PCT with lost tubular architecture in the form of extensive rarefaction (R) of the cytoplasm, loss of the apical microvilli (arrowheads), reduction of number of mitochondria (arrows) with complete loss of their cristae in a rat of group I (3000x). D- PCT with rounded midpositioned nuclei $(\mathrm{N})$, mitochondria $(\mathrm{m})$, thin regular tubular basement membrane (arrows), intact apical microvilli $\left(^{*}\right)$ and lipid droplets (LD) in a rat of group II (3000x).

Both groups III and IV had focal areas of increased interstitial fibrous tissue formation, a thick parietal layer of both Bowman's capsules and tubular basement membranes. These findings were more obvious in group III compared to group IV (Figure 3).

\section{Results of electron microscopy study}

Thickening of the glomerular basement membranes and effacement of the foot processes of the podocytes obliterating slit diaphragms between were observed in group I compared to the control group. In the rats concomitantly treated with verapamil (group II), the glomerular basement membranes became normal but the effacement of the foot processes of the podocytes persisted. Finally, loss of the trilaminar appearance of the glomeru- lar basement membranes with fusion of the foot processes of the podocytes was observed in group IV and to a lesser extent in group III.

In both groups I and III, the PCT had irregular nuclear membranes with margination of chromatin material and loss of their tubular architecture in the form of extensive rarefaction of the cytoplasm, loss of the apical microvilli, and reduction of the number of mitochondria with complete loss of their cristae. A marvellous improvement occurred in group II (after adding verapamil) compared to group I. On the other hand, there were residual pathological findings in group IV (indentation of the nuclei, interruption of the apical microvilli, loss of the mitochondria cristae and myeloid bodies) when compared to group III (Figures 4, 5). 

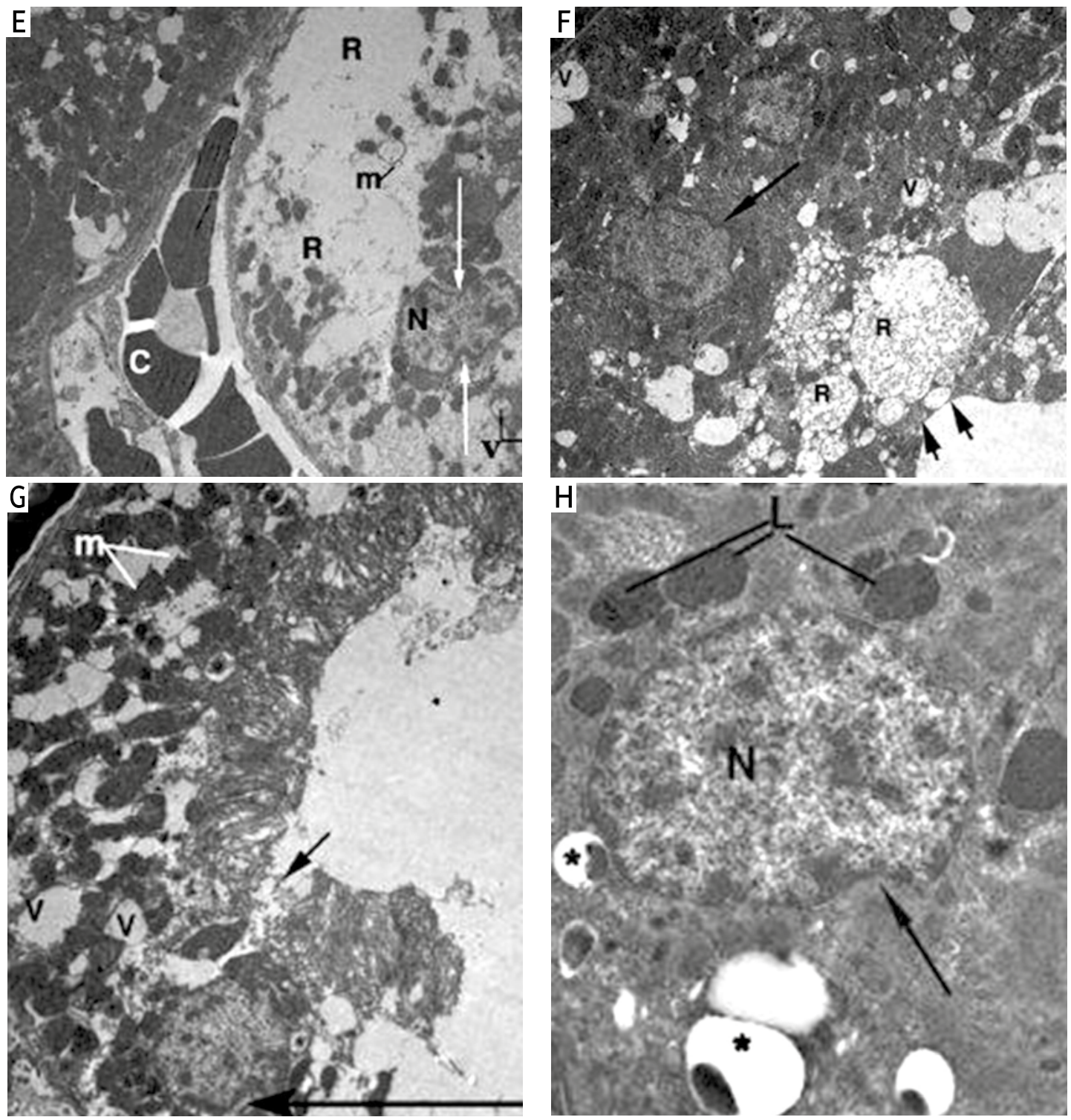

Figure 5.E - PCT with capillary congestion (C), rarefaction of the cytoplasm (R), shrinkage and indentation of the nucleus (arrow), apical vacuoles (V) and loss of mitochondrial $(\mathrm{m})$ cristae in a rat of group III (2500x). F - PCT with loss of the apical microvilli (short arrows), rarefaction (R), vacuolations (V) of the cytoplasm and irregular nuclear membrane (long arrow) in a rat of group III (3000x). G - PCT with indentation of the nucleus (long arrow), interruption of the apical microvilli (short arrow), large vacuoles $(\mathrm{V})$ and loss of cristae of some mitochondria $(\mathrm{m})$ in a rat of group IV (6000x). H - PCT with nuclear indentation (arrow), large lysosomes (L) and myeloid bodies $\left(^{*}\right)$ in a rat of group IV (2500x)

\section{Morphometric results}

The mean area \% of fibrosis of both treated groups I and II increased significantly in comparison with the control group. Much improvement occurred in group II when compared to group I. A statistically insignificant decrease in area \% of collagen was observed in group IV in comparison to group III.

The mean optical density of PAS reaction of both treated groups I and II decreased significantly in comparison with the control group. Much improvement occurred in group II when compared to group I. Nonsignificant changes were observed in groups III and IV.
The mean area of the urinary space of group I increased significantly in comparison with the control group. A statistically insignificant increase was observed in group II when compared to the control group. Insignificant improvement occurred in group II when compared to group I and also in group IV when compared to group III.

The most sensitive measurable parameter was the mean optical density of PAS reactions as its $F$ ratio was 44.4 . On the other hand, the least sensitive parameter was the mean area of the urinary spaces as its $F$ ratio was 1.6 (Tables I and II). 


\section{Discussion}

The kidneys are the primary target organs of mercury accumulation and toxicity in both animals and humans. The most sensitive part of the kidney to the toxic effects of mercuric chloride is the epithelium of PCT. This could be attributed to wide distribution of the enzymes needed for the uptake of mercury in the cells of the PCT [18].

Chronic administration of mercuric chloride resulted in cortical nephrotoxic effects in the form of glomerular sclerosis, acute tubular necrosis and interstitial inflammatory cellular infiltration which eventually ended in interstitial fibrosis. Glomerular affection was observed in many pathological forms. This variability in the pathological effects might be due to the difference in the susceptibility of the glomerular cells to the injurious effects of mercury [19]. The glomerular congestion might be due to the direct injurious effect of the mercuric chloride on the cells of the capillary wall [20]. The shrinkage of the glomeruli with secondary widening of the urinary spaces and thickening of the parietal layer of Bowman's capsule in this group might be a consequence of the glomerular sclerosis [21] or oxidative stress of the mercuric chloride on the kidney [22]. Thickening of the afferent arterioles might be attributed to deposition of the immune complexes on the basement membrane of the renal arterioles causing membranous glomerulonephritis [23]. Effacement of the foot processes of the podocytes could be attributed to an immunological reaction caused by T-lymphocytes and interleukins [24].

Chronic administration of mercuric chloride also affected the renal tubules. Vacuolation in the cells of the PCT could be due to the disturbance of the sodium pump which results in hydropic degeneration [25]. The intraluminal exfoliation might be due to the development of renal ischemia which induces oxidative stress with subsequent formation of oxygen free radicals that attack the renal tubular cells $[18,23]$. Complete loss of the apical brush border might be due to the binding of some antibodies to the antigenic component of the brush border of the PCT [26]. Thickening of the tubular basement membranes is mostly due to deposition of immune complexes as mercury can increase the production of autoantibodies, inflammatory cytokines and cytotoxic T cells which attack the basement membrane of the PCT [27].

Another finding in group I was the presence of interstitial inflammatory cellular infiltration in the renal cortex which might be an immunologic mechanism or renal ischemia induced by the exposure to mercuric chloride [24]. The interstitial exudation that was detected in this group might be caused by release of catecholamines causing vasoconstriction and reduction of the renal blood flow with subsequent tubular damage and leakage of the fluid exudate into the interstitium [28].
Concomitant use of verapamil with mercuric chloride (group II) improved the previous mentioned pathological changes. Such protection was not complete as there was still slight congestion, shrinkage of some glomeruli and decrease in the thickness of the parietal layer of Bowman's capsule. The partial protective effect of verapamil on the glomerular cells might be attributed to its antioxidant effect [29] against oxidative stress of the mercuric chloride. Verapamil inhibits both the formation of more free radicals and the rate of lipid peroxidation and could augment the activities of some renal antioxidant enzymes [30].

Verapamil protects the mitochondria of the renal tubular cells by opposing the inhibitory effect of mercury on the renal mitochondrial enzymes [31]. It also reduces the cellular apoptosis of renal tubular epithelial by being a calcium channel blocker, preventing an increase in the intracellular calcium influx which causes apoptosis of the renal tubular cells [32].

Finally, concomitant administration of verapamil with mercuric chloride resulted in disappearance of the interstitial exudate and reduction in the inflammatory cell infiltration, which might be through improving the renal blood flow due to its vasodilator effect [33].

The aim of both groups III and IV was to compare the reversibility of the toxic effect of mercuric chloride after its withdrawal (group III) with the reversibility after administration of verapamil as a therapeutic agent (group IV).

The pathological findings in groups III and IV were more or less similar, although these findings were less severe in group IV compared to group III. The persistence of the pathological findings in these groups reflects the irreversible nephrotoxic changes caused by chronic mercuric chloride exposure. The use of verapamil after mercuric chloride attenuated the toxic effects of mercuric chloride on the renal cells slightly more than the mercuric chloride withdrawal did alone.

In conclusion, the concomitant administration of verapamil has a much better effect in minimizing the nephrotoxic effect caused by chronic mercuric chloride than its therapeutic administration. So, we recommend the prophylactic use of verapamil in suspected cases of chronic mercuric chloride nephrotoxicity to preserve renal function.

\section{Conflict of interest}

The authors declare no conflict of interest.

\section{References}

1. Farahat SA, Rashed LA, Zawilla NH, Farouk SM. Effect of occupational exposure to elemental mercury in the 
amalgam on thymulin hormone production among dental staff. Toxicol Ind Health 2009; 25: 159-67.

2. Rashed MN. Monitoring of contaminated toxic and heavy metals, from mine tailings through age accumulation, in soil and wild plants at Southeast Egypt. J Hazard Mater 2010; 178: 739-46.

3. Zalups RK. Molecular interactions with mercury in the kidney. Pharmacol Rev 2000; 52: 113-43.

4. Dye BA, Schober SE, Dillon CF, et al. Urinary mercury concentrations associated with dental restorations in adult women aged 16-49 years: United States, 19992000. Occup Environ Med 2005; 62: 368-75.

5. Li SJ, Zhang SH, Chen HP, et al. Mercury-induced membranous nephropathy: clinical and pathological features. Clin J Am Soc Nephrol 2010; 5: 439-44.

6. Bhan A, Sarkar NN. Mercury in the environment: effect on health and reproduction. Rev Environ Health 2005; 20: 39-56.

7. Carranza-Rosales P, Said-Fernandez S, SepulvedaSaavedra J, Cruz-Vega D, Gadolfi A. Morphologic and functional alterations induced by low doses of mercuric chloride in the kidney OK cell line: ultrastructural evidence for an apoptotic mechanism of damage. Toxicology 2005; 210: 111-21.

8. Johansen P, Mulvad G, Pedersen H, Hansen J, Riget F. Human accumulation of mercury in Greenland. Sci Total Environ 2007; 377: 173-78.

9. Xiaolei J, Eric L, Don C, et al. Dietary fats altered nephrotoxicity profile of methyl mercury in rats. J Appl Toxicol 2009; 29: 126-40.

10. Bridges C, Zalups R. Molecular and ionic mimicry and the transport of toxic metals. Toxicol Appl Pharmacol 2005; 204: 274-308.

11. Sakamoto M, Ikegami N, Nakano A. Protective effects of $\mathrm{Ca}^{+2}$ channel blockers against methyl mercury toxicity. Pharmacol Toxicol 1996; 78: 193-9.

12. Yamashita J, Itoh M, Kuro T, Kobayashi Y, Ogata M. Pre or post-ischemic treatment with a novel $\mathrm{Na} / \mathrm{Ca}$ exchange inhibitor, KB-R7943, shows renal protective effects in rats with ischemic acute renal failure. JPET 2001; 29: 412-9.

13. Girardi G, Elias M. Verapamil protection against mercuric chloride-induced renal glomerular injury in rats. Toxicol Appl Pharmacol 1998; 152: 360-5.

14. Shi JK, Kang F, Wu Q, Lu YF, Liu J, Kang YJ. Nephrotoxicity of mercuric chloride, methyl mercury and cinnabarcontaining Zhu-Sha-An-Shen-Wan in rats. Toxicol Lett 2011; 200: 194-200.

15. Lee S, Park J, Yu E, Yang W, Kim S. Individual or combined effects of enalapril and verapamil in chronic cyclosporine nephrotoxicity in rats. J Korean Med Sci 1999; 14: 653-8.

16. Bancroft JD, Gamble M. Theory and practice of histological techniques. Churchill Livingstone. London, Edinburgh, New York, Philadelphia, St Louis, Sydney and Toronto 2002; 167-70.

17. Dykstra MJ, Reuss LE. Biological electron microscopy: theory, techniques, and troubleshooting. Library of Congress, New York 2000.

18. Adriana M, Ankur V, Dnyanmote K, Bush W, Sanjay K. Deletion of multi-specific organic anion transporter protects from mercury-induced kidney injury. J Biol Chem 2011; 286: 1-13.

19. Zalups R. Molecular interactions with mercury in kidney. Pharmacol Rev 2000; 52: 113-43.

20. Kumar V, Fausto N, Abbas A, Mitchell R. Acute and chronic inflammation. In: Robbins basic pathology. Elsevie Saunders, USA 2007; 33-4.

21. Detwiter RK, Falk RJ, Hogan SL, Jennette JC. Collapsing glomerulopathy: a clinical and pathological variant of focal glomerulosclerosis. Kidney Int 1994; 45: 1416-24.
22. Barisoni L, Madai MP, Eraso M, Gasser DL, Nelson PJ. The $\mathrm{kd} / \mathrm{kd}$ mouse is a model of collapsing glomerulopathy. J Am Soc Nephrol 2005; 16: 2847-51.

23. Christian S, Rune D, Pall L. Are liver and renal lesions in East Greenland polar bears (Ursus maritimus) associated with high mercury levels? Environ Health 2007; 6: 11-8.

24. Alwin $\mathrm{H}$, Arthur H. Drug-induced kidney disease. Pathology and current concepts. Annals Academy of Medicine 2009; 38: 240-50

25. Therien A, Blostein R. Mechanisms of sodium pump regulation. Am J Physiol 2000; 279: 541-66.

26. Michel D, Kelly C. Acute interstitial nephritis. J Am Soc Nephrol 1998; 9: 506-15.

27. Nielsen E, Couser W. Immunologic renal diseases. Lippincott-Raven, New York, Philadelphia 2001; 899-929.

28. Humes D, Mackay S, Funke A, Buffington D. Acute renal failure: growth factors, cell therapy and gene therapy. Proc Am Assoc Physicians 1997; 109: 547-57.

29. Xu B, Xu ZF, Deng Y, Yang JH. Protective effects of Chlorpromazine and Verapamil against cadmium-induced kidney damage in vivo. Exp Toxicol Pathol 2010; 62: 27-34.

30. Kedziora-Kornatowska K, Szram T, Kornatowski L, Szadujkis-Szadurski J. The effect of verapamil on the antioxidant defense system in diabetic kidney. Clin Chim Acta 2002; 322: 105-12.

31. Sukru O, Suro C, Sakip E, Ahmet E, Faruk E, Ramazan U. Verapamil prevents the apoptotic and hemodynamic changes in response to unilateral ureteric obstruction. Int J Urol 2008; 15: 350-5.

32. Nagao K, Nishil D, Yoshihara H, et al. Calcium channel inhibition accelerates polycystic kidney disease progression in the rat. Kidney Int 2008; 73: 269-77.

33. Ali B, Al-Oarawi A, Mousa $H$. The effect of calcium load and the calcium channel blocker verapamil on gentamycin nephrotoxicity in rats. Food Chem Toxicol 2002; 40: $1843-74$. 